\title{
Essais
}

Revue interdisciplinaire d'Humanités

Hors-série 1 | 2013

L'estrangement

\section{Rendre étrange le passé récent : la discipline historique dans la tourmente mémorielle espagnole}

François Godicheau

\section{OpenEdition}

Journals

Édition électronique

URL : http://journals.openedition.org/essais/2322

DOI : 10.4000 /essais. 2322

ISSN : 2276-0970

Éditeur

École doctorale Montaigne Humanités

Édition imprimée

Date de publication : 15 octobre 2013

Pagination : 129-145

ISBN : 978-2-9544269-7-4

ISSN : $2417-4211$

Référence électronique

François Godicheau, «Rendre étrange le passé récent : la discipline historique dans la tourmente mémorielle espagnole», Essais [En ligne], Hors-série 1 | 2013, mis en ligne le 05 octobre 2020, consulté le 10 décembre 2020. URL : http://journals.openedition.org/essais/2322 ; DOI : https:// doi.org/10.4000/essais.2322 


\title{
Rendre étrange le passé récent : la discipline historique dans la tourmente mémorielle espagnole
}

\author{
François Godicheau
}

Dans l'Espagne actuelle et depuis une quinzaine d'années, les historiens sont confrontés à une mise en cause de leur travail, de la place de ce travail dans la société ; une mise en cause à la fois explicite, à travers des actions ou déclarations d'autres acteurs sociaux parlant du passé et implicite, liée à leur situation peu avantageuse sur le marché des discours sur le passé. On peut analyser cette situation en faisant jouer l'opposition entre une histoire naturalisatrice et une histoire de l'estrangement. La tourmente mémorielle qui agite depuis une dizaine d'années ce pays illustrerait ainsi la nécessité de pratiquer une histoire visant la distanciation vis à vis des subjectivités d'hier et faisant de l'historien celui qui, de ses voyages dans le passé, ramène de l'étrange pour permettre aux citoyens du présent de prendre leurs distances vis à vis d'eux-mêmes. En un mot, peut-on et doit-on écrire une histoire qui nous dise ce que nous ne sommes pas?

La mise en cause du travail de l'historien dans la société espagnole de ces dernières années peut se décliner en trois volets.

La première mise en cause est la plus directe : celle de journalistes néofranquistes qui taxent le travail des historiens de mythologie et lui opposent une Vérité majuscule, quand en réalité, ils ne font que reprendre dans un style actuel la propagande franquiste des années trente à cinquante, en l'illustrant à l'aide de documents trouvés dans les archives. Depuis la fin des années 1990 en effet, sont apparus des auteurs comme Pío Moa, auteur de plusieurs ouvrages, dont le premier, Les mythes de la guerre civile, a bénéficié d'une très large promotion dans des médias sympathisants avec le parti de droite majoritaire et s'est vendu à plus de 100000 exemplaires. Renversant la charge de la preuve, il qualifiait l'ensemble du travail des historiens universitaires depuis la transition à la démocratie de "mythe ", c'est-à-dire, pour lui, de récit mensonger consistant à charger Franco de tous les maux et à protéger les gauches des années trente qualifiées peu ou prou d'ennemies de la démocratie ${ }^{1}$.

1 Los mitos de la guerra civil, Madrid, La esfera de los libros, 2003. Un autre auteur prolifique, 
La réaction des historiens a consisté à refuser de discuter avec des " amateurs ", mais comme ces " amateurs " passent pour des historiens auprès du grand public et ont vendu des centaines de milliers d'exemplaires, de rares collègues sont malgré tout entrés dans l'arène. Cependant, leur défense a essentiellement consisté à affirmer leur objectivité face aux attitudes partisanes et à combattre les interprétations vicieuses de ces ouvrages néo-franquistes en produisant force nouveaux documents. Les effets ont été limités car les raisons du succès de Pio Moa et consort n'étaient pas tant liées à la qualité de leurs sources primaires qu'à la persistance d'une tradition politique d'interprétation de la guerre dont l'existence renvoie à la manière dont s'est réalisée la transition après la mort de Franco ${ }^{2}$. La dénonciation des mythes franquistes et de leur présence renouvelée dans l'Espagne actuelle, en se limitant à la contraposition de la vérité historique au récit mystificateur, a élevé une digue tout à fait nécessaire face à l'argumentation néo-franquiste mais n'a pas pu faire beaucoup plus que de prêcher les convaincus, tant il est vrai que le mythe est beaucoup plus qu'un récit mensonger.

La deuxième mise en cause est moins directe mais de très longue portée. Elle est le corollaire d'un mouvement social qui a pris le nom de « mouvement de récupération de la mémoire historique " et s'est attaqué aux politiques de mémoire du franquisme et de la monarchie (célébration des morts franquistes, silence sur les autres, présence des symboles franquistes dans l'espace public, absence de symboles antifranquistes). À partir du milieu des années 1990, des associations sont nées pour revendiquer l'héritage des combattants antifranquistes et des victimes de la dictature et dénoncer le traitement symbolique et politique qu'avaient reçu les défenseurs de la démocratie depuis la fin des années trente, pendant le franquisme et les vingt premières années de la monarchie constitutionnelle. Fut ainsi dénoncé le " pacte de silence " que les principaux partis politiques auraient passé à propos de la guerre de 1936, et que le Parti socialiste aurait appliqué pendant quatorze ans. Le contraste terrible entre la présence dans l'espace public démocratique de monuments et de plaques à la gloire du dictateur et de ses hommes, et l'absence complète de ses victimes - dont plus d'une centaine de milliers dans des fosses communes où leurs restes gisent toujours - a été, et est toujours, une des motivations principales de ce qui est très vite devenu une mobilisation politique de grande ampleur. Plusieurs générations se sont en effet engagées, et en particulier celle des petits-enfants de la guerre, dans un activisme mémoriel qui s'est traduit par l'organisation de cérémonies du souvenir et d'activités de vulgarisation, des déclarations politiques, inaugurations de plaques et de monuments, et des exhumations avec recherche d'identification à partir de nombreuses fosses communes retrouvées.

César Vidal, a exploité la même veine, notamment sur les brigades internationales.

2 Les principaux historiens ayant polémiqué avec Pío Moa et autres auteurs ont été Enrique Moradiellos, Alberto Reig Tapia, Francisco Espinosa et dans une moindre mesure Angel Viñas et Paul Preston. 
Comme on s'en doute, très tôt, ce mouvement a interpelé le monde des historiens. Parler de pacte de silence sur la guerre pendant la transition et depuis, alors que de nombreux historiens avaient publié des centaines de livres, ne constituait pas une critique directe mais donnait une idée assez nette de l'importance conférée à ces travaux, de leur impact sur la société espagnole. Les chercheurs professionnels ne furent pas associés aux premières initiatives, ou seulement au gré des hasards et des rencontres. La méfiance était réelle à leur égard et ils n'étaient en tout cas pas considérés comme les hérauts de la vérité sur le passé, même si on reconnaissait la qualité de leurs travaux, tout simplement parce que ceux-ci étaient relativement peu utiles pour les objectifs visés ${ }^{3}$.

La réponse des historiens fut par conséquent majoritairement marquée, au début, par de la condescendance, voire du mépris, certains éminents spécialistes insistant à travers conférences et articles de journaux sur l'opposition entre l'histoire, c'est-à-dire la recherche scientifique de la vérité, pour laquelle il fallait de la patience et de l'abnégation, ainsi qu'une sorte de retrait des passions du monde, et la mémoire, toujours confuse, partielle, impressionniste, mêlant les époques, la raison et l'émotion ${ }^{4}$. Beaucoup de ces réactions ressemblaient à la défense d'un territoire que l'on sentait tout à coup menacé et insuffisamment valorisé par des citoyens dont la dénonciation du "silence » était la preuve qu'ils entraient rarement dans les librairies pour acheter des ouvrages d'histoire, pourtant disponibles en grand nombre 5 . L'incompréhension de certaines plumes pour la demande sociale qui se faisait jour était très nettement corrélée avec leur place dans l'édification d'un récit national qui trouvait dans la démocratie contemporaine son heureuse conclusion, mais elle traduisait aussi la nouveauté des interrogations sur les questions de mémoire, la notion de politiques de mémoire et la place des historiens dans les discours publics sur le passé6.

3 J'ai pu mesurer en 1998 cette méfiance à l'égard des universitaires, lors d'une rencontre de l'association AGE à Madrid, association dont le nom complet résume à lui seul le mélange des objets et des démarches : "Archive Guerre et Exil, pour la création d'un centre d'archives de la Guerre Civile, des Brigades Internationales, des enfants de la Guerre, de la Résistance et de l'Exil Espagnol ». Les raisons de cette méfiance, telles qu'elles m’étaient exposées, alors que, doctorant, j’avais une position d'extériorité relative au monde de "la historia académica " (universitaire) renvoyaient à l'idée d'une grande frilosité des historiens universitaires par rapport aux conditionnements officiels de leurs recherches (ouverture ou fermeture des archives) et aux questions de mémoire. Seuls trois universitaires y participaient : Alicia Alted Vigil, principale historienne de l'exil espagnol de 1939, Pelaï Pagès, spécialiste de l'histoire du POUM, et Enrique Moradiellos, qui fut ensuite le premier à s'engager dans la polémique avec Pio Moa.

4 Sebastiaan Faber, "The Debate about Spain's Past and the Crisis of Academic Legitimacy: the Case of Santos Juliá", The Colorado Review of Hispanic Studies, vol. 5, 2007, p. 165-170.

5 Cet argument a été celui du grand historien Santos Julià : les livres sont disponibles, il n'est que de les lire.

6 Cette nouveauté nous est apparue très nettement en mars 2002 lors du premier colloque organisé en Espagne sur les politiques de mémoire et l'écriture de l'histoire, colloque organisé à la Casa de Velázquez avec la participation de l'Institut d'Histoire du Temps Présent. Les 
Depuis, voyant qu'il y avait là non pas un courant intellectuel proposant un débat polémique mais un mouvement social posant un problème politique de rapport au passé, la plupart des historiens ont incorporé les questions de mémoire, mais simplement comme un thème supplémentaire à leur agenda de publications, sans toujours réfléchir aux implications sur la démarche au cœur de leur travail. "La mémoire " est même devenue un domaine porteur, mais presque comme un terrain de plus; et même si des contributions au débat de grande qualité ont été produites, tant individuelles que collectives, l'écriture de l'histoire de la guerre de 1936, méthodologiquement très conservatrice, n'a pratiquement pas évolué ; elle a simplement intégré les questions mémorielles comme une sorte d'apostille, demande sociale oblige.

La situation actuelle des historiens en Espagne par rapport à l'histoire du $\mathrm{XX}^{\mathrm{e}}$ siècle est loin d'être unique ; elle est simplement plus incommode qu'ailleurs. La difficulté à construire des objets en fonction de l'outillage intellectuel propre à la discipline historique - considérée dans son ensemble et non pas seulement dans sa version contemporanéiste - et non pas en fonction des sollicitations des entreprises de mémoire, étatiques, médiatiques ou autres, est, en histoire contemporaine, une constante. Le poids des discours publics sur le passé et des catégories qui y sont employées y est plus fort et uniforme qu'ailleurs, ce qui devrait mettre à l'ordre du jour une réflexion permanente sur la place des historiens et de leur travail dans l'articulation des différents régimes d'historicité et dans les multiples discours publics sur le passé. Dans le cas espagnol, l'importance capitale des politiques de mémoire franquistes dans la mise en cuvre de la transition et dans la vie politique de l'actuelle monarchie fait des questions du rapport au passé un enjeu politique majeur, souligné encore par les diverses expressions politiques de la crise économique et sociale actuelle. Le débat politique incorpore beaucoup plus qu'hier des références au passé récent, que ce soit chez les partis nationalistes ou chez les grands partis de gouvernement. Ceux-ci cherchent à réaffirmer leur identité à l'heure où la ressemblance de leurs politiques économiques et sociales tend à les confondre. Comme dans plusieurs pays voisins, cela est lié à leur acceptation partagée des grandes directions de la politique économique et sociale de l'Union Européenne, mais en Espagne, cela s'explique aussi par une recherche

actes ont été publiés quatre ans après : Julio Aróstegui Sánchez et François Godicheau (éd.), La guerra civil. Mito y memoria, Madrid, Marcial Pons, 2006. La notion de " politiques de mémoire " était relativement inconnue dans l'Espagne de ce moment (elle commença à être diffusée seulement avec le livre de Stéphane Michonneau, Barcelona, memoria e identitat. Monuments, conmemoracions $i$ mites, chez Eumo, au printemps de cette année 2002). On put mesurer lors de cette rencontre la distance entre la réflexion sur l'histoire du temps présent en France, à travers le travail de l'IHTP et notamment les ouvrages d'Henry Rousso, ou sur la sociologie de la mémoire, avec les publications de Marie-Claire Lavabre, et l'état des réflexions en Espagne où Julio Aróstegui était presque le seul, à l'Université Complutense de Madrid, à s'engager dans cette voie. 
presque systématique de consensus et de pactes entre gouvernement et opposition, ce qui est une marque de fabrique de la monarchie constitutionnelle depuis 1978.

Les historiens de la guerre civile, habitués qu'ils étaient à être les seuls experts reconnus de ce passé, sont devenus des auteurs presque " comme les autres ", parmi tous ceux qui écrivent sur la question ; et le comble est que le public préfere souvent à leurs livres des récits fictionnels plus ou moins hybrides, ceux que l'on appelle pour l'Espagne les « romans de la mémoire ». Ceux-ci, apparus par centaines depuis deux décennies, jouent avec la référentialité et les sources documentaires de sorte à brouiller la frontière avec les essais historiques ${ }^{7}$. Les historiens doivent aussi, beaucoup plus que naguère, partager l'attention du public et les étals des librairies avec des récits de mémoire, plus ou moins fictionnalisés, des témoignages, des éditions de documents bruts souvent publiés sans appareil critique ou des documentaires vidéo ; leur spécificité en tant que scientifiques est devenue presque inaudible. Pire, elle s'exprime souvent sous la forme de l'exigence outrée du respect de leur magistère.

Le problème de ces autorités qui cherchent à se faire respecter est que leur crédibilité est durablement entamée et que leur position est parfois difficile à tenir. Leur autorité vient en grande partie de la position dans laquelle ils ont été placés pendant la transition démocratique. Celle-ci en a fait les producteurs et les garants d'un discours pacificateur sur la guerre de 1936, pacificateur en tant qu'ils savaient « faire parler les archives », qu' ils contrôlaient « l'expression objective des documents ", loin de toute interprétation, vue comme nécessairement partisane. Ils furent par exemple chargés officiellement, en 1977, alors que face à l'histoire " républicaine " du bombardement de Guernica, l'idée, inventée par l'état-major franquiste au lendemain des faits, selon laquelle les " rouges " auraient mis en scène le massacre à coups de lance-flammes, tenait toujours, de bâtir une version « objective » en vertu de leur accès aux sources ${ }^{8}$. Celles-ci étaient sensées parler à travers eux sans qu'il fût question de la part interprétative de leur travail, des catégories de leur questionnement ni, encore moins, de faire une analyse critique des versions affrontées l'une à l'autre ni du rapport de ces discours aux faits?

7 Le livre le plus connu de ce nouveau genre est le bestseller de Javier Cercas, Soldats de Salamine (paru en français chez Actes Sud, en 2002), près d'un million d'exemplaires vendus en Espagne. Le romancier Isaac Rosa, qui dans son livre Encore un fichu roman sur la guerre d'Espagne (Bourgois, 2010), critique de façon grinçante cette veine juteuse et ses productions de médiocre qualité, a estimé à plus de 800 le nombre de « romans » publiés depuis vingt ans et qui traitent de cette période.

8 Pour un récit détaillé de cette anecdote, cf. Jesús Izquierdo Martín et Pablo Sánchez León, La guerra que nos han contado, Madrid, Alianza, 2006, p. 57 et suivantes.

9 Pourtant, il existait un magnifique exemple d'une démarche différente avec le livre d'Herbert Southworth, El mito de la cruzada de Franco, qui s'était appliqué, une dizaine d'années auparavant, en 1963, à démonter le discours propagandiste de deux plumitifs de l'Opus Dei 
En même temps, les politiques de mémoire des derniers gouvernements franquistes, des années soixante aux années 1976-1977, fixaient un cadre d'interprétation de la guerre devenu et resté depuis hégémonique, un cadre indispensable à la justification politique de la transition telle qu' elle devait se dérouler : la guerre de 1936 était une " folie collective ", une " guerre fratricide " aux responsabilités et aux horreurs également réparties. Celles-ci appelaient comme une nécessité la réconciliation nationale, le "plus jamais ça ", le regard tourné vers l'avenir, la marginalisation politique de tout discours républicain et enfin (et surtout) l'impunité des crimes de la dictature. Cette impunité fut réalisée à travers la loi de 1977 par laquelle le gouvernement, en amnistiant la plupart des prisonniers politiques antifranquistes, amnistiait aussi le personnel de la dictature chargé de la répression depuis la guerre. Ces politiques de mémoire officielles rejoignaient et reprenaient les appels à la réconciliation nationale venant du PCE et du PSOE depuis les années 1950.

Toute l'économie morale de la vie publique de l'actuelle démocratie repose sur le récit de la transition comme naissance d'une nouvelle Espagne, celle de la culture du consensus, du pacte social, du "vivre ensemble pacifique " [convivencia pacifica], du progrès, et donc sur le rejet du passé de guerre civile et l'oubli du franquisme comme dictature, jusqu'au point que l'expression « la dictature » fût euphémisée en "le régime antérieur » dans des manuels scolaires des années $1990 .{ }^{10}$ Le débat politique espagnol est marqué depuis lors par la recherche permanente de " pactes de législature » entre les deux grands partis majoritaires, que ce soit sur la lutte contre le terrorisme d'ETA ou à propos de l'économie du pays. La politique ainsi mise en œuvre ne peut être que «la meilleure politique possible ", rationnelle parce que consensuelle, consensus considéré et présenté comme la marque d'une modernité forcément "européenne " et sanctionnée par une réussite économique " exemplaire " ${ }^{11}$.

Le problème est que vingt ou trente ans après, le tableau de fond du décor transitionnel se déchire : la critique virulente des politiques de mémoire de la transition (condamnation du " pacte de silence ", ouverture des fosses

chargés par le régime d'actualiser le mythe fondateur de la guerre de 1936 comme croisade. Dans ce livre, c'est l'analyse du caractère biaisé de l'argumentation franquiste qui primait et l'auteur, en bon historien, ne chargeait par les archives de démonter seules le faux. La différence avec la mission confiée aux historiens à propos de Guernica venait de la vision que les responsables politiques avaient de la discipline historique, même s'il ne faut pas négliger le souci de fuir le débat contradictoire sur le passé.

10 Ce dernier fait fut l'ultime déclencheur de la colère de deux vieux et importants militants antifranquistes Nicolás Sartorius et Javier Alfaya, qui publièrent en 1999 un des premiers livres de revendication mémorielle, qui fut un succès de librairie : La Memoria Insumisa. Sobre la dictadura de Franco (Madrid, Espasa, Calpe).

11 Sur les rapports entre l'idée de consensus et l'évolution politique de l'Espagne à ce moment, voir deux livres essentiels : Bénédicte André Bazzana, Mitos y mentiras de la transición, Barcelone, El Viejo Topo, 2006 ; et Joan Garcès, Soberanos e intervenidos. Estrategias globales, americanos y españoles, Madrid, Siglo XXI, 2012. 
communes) rejoint les critiques plus politiques des insuffisances de la démocratie actuelle. Cette critique prend des formes diverses, la plus connue étant le mouvement dit du 15 mai 2011 - connu en France sous le nom de "mouvement des indignés " - et avec des revendications qui varient de l'exigence de la proportionnelle à celle d'une III ${ }^{e}$ République en passant par la défense de la santé et de l'éducation face aux coupes budgétaires. Mais surtout, la vision d'eux-mêmes que les ténors politiques avaient vendu aux Espagnols est entrée dans une crise profonde depuis que le miroir de la croissance économique exemplaire s'est brisé ${ }^{12}$. Un chômage sans précédent $-27 \%$ de la population active, $57 \%$ chez les jeunes en avril 2013 -, le développement de la xénophobie, la corruption généralisée qui s'étale dans les journaux et le fait que la politique consistant à sauver les banques en demandant toujours plus de sacrifices aux citoyens soit imposée par cette même Europe qui incarnait l'idée même de modernité, tout cela contribue fortement à ruiner le " consensus » transitionnel sur le passé récent.

Pendant ce temps, les deux grands partis de droite et de gauche, tout en cherchant toujours à incarner la seule position légitimée par la transition, c'est-à-dire le centre politique, revendiquent de plus en plus clairement des signes d'identités qu'ils vont chercher dans le passé antifranquiste pour les uns, franquiste pour les autres ${ }^{13}$. Au moment où des non-professionnels écrivent et vendent force livres d'histoires, où eux aussi prétendent " faire parler les archives " (accessibles à tous), ou bien des ouvrages où les archives " parlent toutes seules ", on assiste donc à l'écroulement de l'idéologie du progrès qui a sous-tendu l'écriture de l'histoire du XX' siècle espagnol durant ces trente dernières années, une histoire dure mais rachetée par sa fin heureuse, celle de la transition et de l'intégration à l'Europe. L'effondrement de cette idéologie dans un des derniers pays d'Europe occidentale où elle fleurissait encore entraîne à sa suite la belle hiérarchie qui faisait de nos grands-parents des versions imparfaites de nous-mêmes. Dans ce contexte, les historiens du XXe siècle espagnol, dont les narrations ont été encadrées depuis plusieurs décennies dans le métarécit d'un progrès ininterrompu, sont bien en peine d'aider les citoyens à faire progresser le débat public. Ballotés entre la défense de telle option politique et le retrait dans la tour d'ivoire de l'objectivisme, la résidence où on les a assignés depuis trente ans, ils sont en

12 Un des symptômes de cette perte d'efficacité du modèle identitaire dominant est le succès du livre dirigé par Guillém Martínez (éd.), CT. Cultura de la transición. Critica a 35 años de cultura española, Barcelona, Debolsillo, 2012.

13 L'affrontement symbolique était spectaculaire autour de la figure du chef du gouvernement José Luis Rodríguez Zapatero, qui revendiquait la mémoire de son grand-père, Garde civil républicain fusillé par les franquistes, et auxquels des manifestants d'opposition chantèrent plusieurs fois " Zapatero, dans la fosse, comme ton grand père!" ("Zapatero, al hoyo, igual que tu abuelo »). Dernièrement, le 13 mai 2013, la déléguée du gouvernement central en Catalogne a créé le scandale en décernant un diplôme à un représentant de la División Azul, la division de volontaires que Franco avait envoyée combattre aux côtés de l'armée d'Hitler sur le front russe. 
réalité bloqués dans leur possibilité de réagir par ce qu'ils partagent avec leurs adversaires et concurrents : la tendance à naturaliser le passé et en particulier les subjectivités passées.

\section{Naturalisation du passé}

On peut prendre comme illustration de cette naturalisation un livre collectif intitulé Palabras como puños (des mots comme des poings) dont le sous-titre est " une histoire de l'intransigeance politique "; un livre qui opère une lecture complètement téléologique et anachronique des années 1930 à partir de l'idée d'intransigeance politique ${ }^{14}$. Ce défaut moral, affectant d'abord les « extrêmes " mais contaminant - ô malheur ! - les partis modérés, est finalement ce qui expliquerait l'embrasement de l'Espagne en 1936 et accessoirement la persistance de la violence politique dans l'Espagne démocratique depuis 1978. En contrepoint, on trouve évidemment la tolérance et la capacité de dialogue qui caractériseraient l'Espagne d'aujourd'hui.

L'attitude d'ensemble de la profession relève d'une manière générale d'une recherche de l'équilibre et consiste à faire le tri entre ce qui, parmi les attitudes des acteurs passés peut être revendiqué et ce qui doit être condamné, le tout reposant sur une familiarisation du passé : les subjectivités des acteurs passés sont censées être les mêmes que celles d'aujourd'hui, ils sont censés obéir à nos critères de rationalité ${ }^{15}$. On va alors "récupérer » ce que nous aurions perdu et dont la démocratie d'aujourd'hui manquerait, le passé se réduisant alors à une sorte de vieux supermarché où l'on va chercher les produits que l'on connaît mais qui auraient disparu de nos étals.

Cette familiarisation procède à la fois d'une réification des identités collectives de l'époque et d'un intérêt très faible pour les sujets individuels. Dans les histoires de la guerre de 1936, on trouve d'abord et surtout des sujets collectifs réifiés (les masses, les anarchistes, les républicains, les communistes, etc.), des "blocs " idéologiques ou sociaux homogènes et même opaques. En revanche, les individus sont complètement absents de ces récits, sauf quelques figures éminentes, dirigeants politiques, syndicaux, militaires, sauf parfois aussi comme illustrations de phénomènes ou d'actions dont les véritables auteurs sont " les ouvriers", " les paysans sans terre ", " la bourgeoisie ", " la CNT ", "les républicains ", "les phalangistes ", etc. Ces illustrations ne pensent pas. La plupart du temps, elles ne parlent pas, et quand elles le font, elles s'expriment comme nous : l'historien projette sur ces sujets qui agrémentent son récit sa propre rationalité, ses mots et son monde (un monde où "intransi-

14 Fernando del Rey Reguillo (éd.), Palabras como puños. La intransigencia política en la segunda República española, Madrid, Tecnos, 2011.

15 Jesús Izquierdo Martín et Pablo Sánchez León, op. cit., p. 149 et suivantes. 
geance politique " a un sens certain). Certains historiens, comme l'américain Michael Seidman, ne se contentent pas de projeter une rationalité ou une subjectivité du présent vers le passé ; ils vont jusqu’à projeter une rationalité utilitariste, c'est-à-dire jamais vérifiée, imaginaire. Pour Seidman, qui ne distingue que deux types anthropologiques, celui du militant disposé à sacrifier sa vie pour une cause, et celui de l'individu normal - les autres, très majoritaires -, la plupart de sujets de l'époque ont avant tout cherché à minimiser leurs pertes et à maximiser leurs profits. Ce schématisme réduit le champ de l'engagement politique à une marge peuplée de fanatiques et fait de la plupart des Espagnols de 1936 des victimes d'une situation de guerre qui ne peut dès lors être décrite que comme une "folie furieuse ». La dénonciation des «idéologies ", forcément mortiferes, s'accompagne de la valorisation d'une logique sociale, censée être celle de notre présent, où le libre jeu des calculs d'intérêts individuels, une fois disparues les idéologies, apporterait l'harmonie ${ }^{16}$.

Un critique de cette naturalisation parle à ce propos de "monologue des vivants " : les morts dans ces conditions n'auraient rien à nous dire de véritablement important ; ils sont dépassés ; ils représentent juste un moment de l'histoire de la réalisation de nous-mêmes, de notre identité pleinement réalisée de démocrates espagnols, européens et prospères ${ }^{17}$. Ils sont finalement réduits à ce que sont les mues pour un serpent vivant, réduits à leur enveloppe : leur subjectivité, disparue et sans doute en grande partie hors d'atteinte, n'est pas objet d'enquête.

Ceux qui dans l'Espagne d'aujourd'hui mettent en cause le travail des historiens, publicistes néo-franquistes ou mouvement de la mémoire, n'échappent pas non plus à cette familiarisation du passé.

Les plumes d'extrême droite considèrent elles aussi la réalité passée comme évidente, immédiatement accessible ; elles mettent le recyclage du récit franquiste de la guerre au service d'une version conservatrice - mais tout aussi légitimatrice - de la transition démocratique. L'originalité principale du récit de Pio Moa et consort a consisté, dans un contexte de forte polémique avec le Parti socialiste, à faire porter au PSOE des années trente la responsabilité de la rupture de la légalité institutionnelle lors de la grève générale insurrectionnelle de février 1934 dans les Asturies. Alors que l'historiographie classique s'entendait pour accuser les anarchistes de la fragilisation de la République du fait de leurs mouvements de grève insurrectionnelle de 1933, le nouveau récit de ces publicistes, en reprenant cette justification franquiste du coup d'État

16 On peut noter que cette conception libérale relativement classique est compatible avec les réflexions plus récentes, sur la violence et les vertus du dialogue, d'un Richard Rorty.

17 Jesús Izquierdo Martín, "Dialogar con los muertos. Juegos especulares entre la historia y la memoria ", in M. Reznik y Colectivo Heterogéneo (éd.), Día y niebla, Terezín, encrucijada de poetas, Buenos Aires, Enargeis, 2012, p. 143-157. 
du 18 juillet 1936 - sur l'air de «nous n'avons pas commencé, il n’y avait plus de régime légal "-, exhumait un argumentaire pseudo légaliste contre "les socialistes ", assimilant ceux d'hier à ceux d'aujourd'hui, en un mouvement de double essentialisation.

Dans ce type de récit, les morts n'ont rien à nous dire non plus. Ils sont simplement là pour donner du poids à une vision intentionnaliste, conspiratrice du passé, qui sert à condamner un ensemble vaguement identifié comme " la gauche ", un ensemble idéologique identifié comme étant une seule et même chose depuis les années trente jusqu'à aujourd'hui. Cela est facilité par le fait que cette identification est presque revendiquée par une partie de l'intelligentsia socialiste d'aujourd'hui, alors que le PSOE de Felipe González avait vécu depuis 1974 et pendant les trois mandatures de celui-ci (1982-1996), le dos tourné à son passé tumultueux des années trente.

Le mouvement de la mémoire met en avant quant à lui les histoires individuelles et collectives des victimes du franquisme. Il compose un récit polyphonique très fortement chargé émotionnellement, une émotion qui repose sur l'identification avec les victimes. Il s'agit de faire exister dans l'espace public la parole des sujets individuels complètement absente des grands récits des historiens, sujets et témoins d'abord considérés en tant qu'ils sont victimes : c'est la douleur personnelle et le traumatisme familial qui s'expriment d'abord. Certaines de ces prises de parole se font au bord des fosses communes lors des cérémonies d'exhumation et font l'objet d'un travail très intéressant de la part d'une équipe d'anthropologues de Madrid. D'autres se font aussi de plus en plus à travers la presse, lors d'inauguration de plaques ou de monuments ou sur des sites internet. L'ensemble constitue une rupture très forte avec des décennies de silence : l'évocation des assassinats, viols, tontes, tortures en tout genre, vols d'enfants était non seulement absente de la place publique pendant la dictature, mais elle fut aussi refoulée pendant la transition, au nom de la réconciliation nationale et du danger qu'il y aurait eu à " provoquer » ainsi les franquistes les plus irréductibles, en particulier au sein de l'armée ${ }^{18}$. Pire, dans l'espace privé, ces mots n'étaient pas les bienvenus car faute de reconnaissance sociale de la souffrance, faute de cadre discursif collectif, la douleur ne trouvait pas à s'exprimer car elle était en quelque sorte inaudible - cela est particulièrement vrai dans le cas des violences exercées par les franquistes contre les femmes -, sans compter qu'il pouvait être très dangereux de parler sous la dictature, si d'aventure un enfant venait à répéter innocemment des bribes de conversation à l'école.

18 Sur ce travail anthropologique mené sous la direction de Francisco Ferrandiz, voir son article en ligne : "Exhumaciones y políticas de la memoria en la Espańa contemporánea ", Hispania Nova, Revista de historia contemporánea, n 7, 2007 : http://hispanianova.rediris.es/7/ dossier/07d003.pdf. 
Ce mouvement de prise de parole en Espagne a fait en outre entrer pleinement ce pays dans « l'ère du témoin " et permet dès lors des analyses comparées sur la place des témoins dans les récits du passé avec des aires géographiques, comme l'Europe centrale et orientale, ou l'Amérique du Sud, où cette dynamique existe depuis de longues années ${ }^{19}$. Mais l'évocation douloureuse des souffrances des victimes peut à la longue se convertir en "monologue des morts " et servir, au présent, à des politiques qui trouvent dans ce passé familiarisé une justification absolue, face à laquelle il n'est pas de dialogue possible. Cela a été le cas naguère avec l'instrumentalisation de la douleur des victimes d'ETA par le Parti Populaire et les associations affines au Pays Basque. Les sujets du passé sont alors réifiés en tant que victimes et plus que l'interrogation sur leur subjectivité, c'est l'affirmation de leur souffrance qui intéresse, mais ils deviennent alors des objets, des projectiles que l'on peut se lancer d'un camp à l'autre ${ }^{20}$. Le débat sur le passé tend à devenir un conflit politique et social d'interprétation où les historiens sont convoqués pour leur capacité à valider des interprétations ou des identifications du passé réalisées en fonction d'objectifs politiques du présent, et souvent d'objectifs politiques institutionnels ou partisans. Cette validation passe la plupart du temps par l'apport documentaire ou, de façon secondaire, par le prestige des différents écrivains sur le marché du livre.

\section{Une nécessaire pratique de l'estrangement}

Le contexte espagnol que je viens d'exposer me semble appeler (à grands cris) une mise en pratique des principes de l'estrangement tels qu'ils se dégagent, article après article, de l'œuvre de Carlo Ginzburg. Dans le premier article de son ouvrage de 1998, on lit que l'estrangement est un antidote efficace contre le risque qui nous guette tous, celui de faire de la réalité une évidence ${ }^{21}$. La potentialité corrosive de l'estrangement dont il est question dans ce texte, si elle devient un parti pris de l'historien, peut être une arme tout à fait intéressante pour que les appropriations du passé à l'œuvre dans l'espace public apparaissent pour ce qu'elles sont, c'est-à-dire des interprétations intéressées. Il s'agit de faire en sorte que les acteurs d'hier nous apparaissent dans toute leur étrangeté, c'est-à-dire leur caractère étranger à nos modes de penser d'aujourd'hui. Cela implique d'aller au-delà des « faux-amis » que sont les mots que nous avons en commun.

Notons à ce titre que l'illusion de proximité avec ces acteurs est d'autant plus grande qu'ils ne nous précèdent que de deux générations et que nous connaissons des vieillards qui ont été ces acteurs du passé. L'étude des textes

19 Cette comparaison a été l'objet d'un séminaire en quatre journées d'études à l'EHEHI de la Casa de Velazquez durant l'année 2012 : http://www.casadevelazquez.org/recherchescientifique/news/le-recit-a-lepreuve-du-passe/.

20 Jesús Izquierdo Martín, op. cit.

21 Carlo Ginzburg, "L'Estrangement. Préhistoire d'un procédé littéraire ", in À Distance. Neuf essais sur le point de vue en histoire (1998), Paris, Gallimard, 2001, p. 15-36. 
et des images que cette époque nous a légués pourrait donc se faire à partir d'une attitude toute en recherche des petits détails, des traces de subjectivités qui ne sont plus les nôtres. Il s'agit bien sûr de la "lecture à rebrousse-poil " mais dans l'interprétation que lui donne Simona Cerutti dans son article paru dans Critique $e^{22}$ : déceler dans la source des contenus qui ne sont pas immédiatement saisissables car relevant de catégories disparues ou changées, et même pousser cette attitude jusqu'aux mots qui nous semblent évidents, en une attitude d'historicisation systématique des concepts ${ }^{23}$. Cette étude à la recherche des petites dissonances qui dénoncent l'étrange peut être aussi, bien sûr, celle de la "lecture lente " que Carlo Ginzburg a préconisée dès ses premiers séminaires ${ }^{24}$. Ce n'est pas en effet parce que certains d'entre eux sont toujours vivants que leur subjectivité d'alors, leur " je " de 1936, n'a pas disparu, recouvert par ce que le temps a produit en eux.

J'aimerais affirmer que tout cela est d'abord une position de principe : il s'agit de refuser, comme le fait le Proust dans l'article cité sur l'estrangement, et comme invite à le faire Carlo Ginzburg en amoureux de la réalité, refuser, donc que connaître signifie s'approprier la réalité, mettre un schéma sur elle ${ }^{25}$. Il s'agit de faire nôtres les doutes du même Proust sur notre capacité à connaître vraiment l'intentionnalité de l'état-major allemand en 1918. Or, on sait l'importance de l'intentionnalisme dans les lectures institutionnelles de l'histoire, dans la présentation d'un passé évident qui nous parlerait et qui exigerait de nous telle ou telle attitude conforme à ce que nous serions " ontologiquement ", une ontologie légitimée par la profondeur du passé. Et il s'agit aussi de rendre compte de cette étrangeté, de rendre étrange ce que nous percevons comme étranger, ce qui implique de réfléchir à une manière d'écrire peut-être expérimentale, qui puisse dé-familiariser une réalité passée que les politiques de mémoires des uns et des autres ne cessent de présenter comme évidente. Cela signifie aussi prendre en compte les habitudes de lecture du public : surprendre le lecteur par une forme inattendue est un des objectifs avoués de l'écriture de l'histoire pratiquée par Carlo Ginzburg. On peut voir cela comme le regard dans un miroir déformant, un miroir dans lequel nous cessions de nous trouver beaux ou de chercher l'image que nous pensons être la nôtre, où nous prenions le risque de nous voir autrement, comme dans ces images d'autrefois d'où le mot grotesque a tiré son sens.

22 Simona Cerutti, «À rebrousse-poil : dialogue sur la méthode », Critique, 2011/6, n 769-770, p. 564-575.

23 L'histoire des concepts devient d'autant plus importante que nous nous rendons compte que les sujets d'hier ont constitué leur subjectivité à travers des relations sociales et une façon de dire le monde qui dépendaient étroitement du dictionnaire qu'ils avaient à leur disposition, du cadre conceptuel qui était celui de leur lieu et de leur époque, cadre qui laisse voir de nombreux cas d'homonymie avec notre propre vocabulaire, mais qui relève d'une valeur, d'un usage et d'une configuration des concepts bien différents des nôtres.

24 Notamment celui qui a donné lieu, avec Adriano Prosperi, à la publication de Giochi di pazienza. Un seminario sul "Beneficio di Cristo", Turin, Einaudi, 1975.

25 Carlo Ginzburg, "L'Estrangement », art. cit. 
À ce titre, une première porte d'entrée pour dé-familiariser la guerre de 1936 peut être celle du mythe. Deux auteurs d'un livre intitulé 1936. La guerre civile et nous, se sont arrêtés sur cette question du mythe et l'ont transformée en un outil d'estrangement en s'appuyant sur sa " capacité à mettre entre guillemets la tradition propre et celle d'autrui " pour reprendre les mots du commentaire sur La forge de vulcain de Velázquez, dans l'article "Mythe " du même ouvrage sur le point de vue en histoire ${ }^{26}$.

Comme je l'ai déjà évoqué, des publicistes néo-franquistes à succès ont accusé les historiens (généralement progressistes) de bâtir un mythe de la guerre de 1936 et ont présenté leurs propres livres comme la vérité, une vérité sensée être "définitive ". Les historiens qui ont daigné répondre ont retourné l'accusation dans les mêmes termes (la vérité contre le mythe) et avec le même style d'argumentation basée sur l'idée que les documents parlent d'eux-mêmes. Ils n'ont en général pas montré (au contraire de ce que d'autres historiens avaient fait dans les années 1960 face à la propagande du régime franquiste) à quel point ce qui péchait était l'interprétation des documents par les publicistes en question; ce ne faisant pas, ils ont tout mis en oeuvre pour masquer le fait que eux aussi, historiens professionnels, interprétaient les documents, évitant ainsi qu'on leur pose des questions sur les fondements de leur interprétation. Nos deux auteurs montrent que ces fondements sont ceux du récit hégémonique de la transition démocratique, récit de la guerre comme folie collective et de la nécessaire réconciliation. On peut appliquer ici le raisonnement que l'on trouve à la fin de l'article "Mythe » déjà cité : identifier ce récit comme un mythe, c'est l'identifier comme récit déjà raconté, récit que l'on connaît, qui est partagé, et qui a en son cœur un nom, ni vrai, ni faux. Ce nom c'est celui de "guerre civile" ou de "guerre fratricide». Ces expressions portent en elles l'interprétation hégémonique de la guerre de 1936. Pour la rendre étrange, il faut s'intéresser non seulement au sens que les mots employés par les acteurs avaient pour eux, mais aussi à la centralité de tel ou tel concept. Or l'emploi de l'expression " guerre civile " est extrêmement minoritaire de 1936 à 1939, il n'est pas certain que son sens soit le même et enfin d'autres concepts permettent de définir la réalité de la guerre d'alors (" croisade ", " révolution »). Aujourd'hui, c'est la critique politique vis à vis de l'ordre hérité de la transition qui fait que ce discours de la « guerre fratricide » n'est plus reçu comme si évident. La naturalisation des sujets passés au service d'une vision des Espagnols conforme à la morale dominante de la transition démocratique se brise contre la réalité de la crise multiforme vécue par le pays aujourd'hui. La porte de l'estrangement est ouverte, des historiens pourraient bien la franchir.

Pour cela, il faudra qu'ils cessent de considérer la distance elle aussi comme une évidence donnée par l'éloignement temporel. La distance n'est pas plus le fruit de cet éloignement que la conséquence de l'accumulation d'un savoir

26 Jesús Izquierdo Martín et Pablo Sánchez León, La Guerra, op. cit. ; Carlo Ginzburg, « Mythe. Distance et mensonge ", in $\grave{A}$ distance, op. cit., p. 37-72. 
objectif et de la neutralité de l'historien. L'historien du XXe siècle a tendance à prétendre compenser le manque de profondeur chronologique par une affirmation de sa neutralité, de la suspension de son jugement. Or l'importance conférée à l'idée même de sa neutralité ou de son parti pris dénonce la familiarité depuis laquelle il considère ce passé. La distance induite par l'estrangement implique un certain regard, une attention aux traces de l'étrangeté, un travail véritable ; elle n'est pas donnée, pas plus qu'elle n'est le résultat d'une ascèse ou d'une fermeté éthique. En plus de relever d'un choix axiomatique de l'historien, elle est le résultat d'un effort de lecture et donc sans doute aussi d'un certain métier. Elle peut viser l'inattendu, comme cette lecture des romans de chevalerie dont il est question dans un autre article de Carlo Ginzburg (dans Le Fil et les Traces) : on peut lire les fables pour dégager des vérités sur les subjectivités du passé27. On peut aussi lire les témoignages d'acteurs du XXe siècle de la même façon. On a là le sens original donné, je crois, à l'idée de lecture des sources à rebrousse-poil : les lire comme autre chose que ce pour quoi elles ont été produites. Or, dans la mesure où, comme le fait remarquer Simona Cerutti dans le même article, une très grande partie de nos sources sont des sources normatives, en particulier les sources juridiques, cette lecture de l'estrangement qui s'impose est une lecture forcément corrosive ${ }^{28}$.

On peut opposer même ces vérités sur les subjectivités du passé à la vérité projetée par les sources normatives au sens où celles-ci ne font pas que décrire la réalité ; leur description est souvent prescription, elle relève des discours performatifs qui ont vocation à s'imposer aux acteurs et notamment à les identifier, c'est-à-dire à les saisir et à les tenir dans un certain ordre discursif qui est aussi un ordre du monde ${ }^{29}$. Leur regard sur le passé, le regard des institutions, est celui de la perspective et c'est sans doute grâce à cela qu'elles soumettent et transforment le monde. La parole des acteurs non institutionnels y est située comme contrepoint à la norme, mais elle est considérée comme n'étant pas véritablement une parole. C'est Jacques Rancière qui exprime cette idée :

Le meilleur exemple en estle Montaillou village occitan d'Emmanuel Le Roy Ladurie. L'historien part des actes du procès. Mais sa question ne porte pas sur le matériel juridique comme tel. Elle n'est pas de savoir si les accusés étaient vraiment hérétiques, les témoignages véridiques ou les transcriptions exactes. Elle est de savoir de quel type de réalité ces témoignages sont l'indice, comment ces gens ont vécu l'hérésie. Et sa réponse est finalement qu'ils ne l'ont pas vécue comme une pensée. Elle n'était pas chez eux une interprétation des textes sacrés au moyen de théologies ou de philosophies quils se seraient appropriées par des voies plus ou moins anormales. Elle était l'expression de leur manière de vivre normale, la projection céleste du

27 "Paris, 1647 : un dialogue sur fiction et histoire ", in Le fil et les traces. Vrai faux fictif, Paris, Verdier, 2010, p. 117-140.

28 Simona Cerutti, «À rebrousse poil », art. cit., p. 569.

29 On peut relire à ce propos le célèbre livre de l'anthropologue Mary Douglas, Comment pensent les institutions, Paris, La Découverte, 2010. 
mode d'être d'une communauté marquée à la fois par la chaleur de l'intimité villageoise et par la distance du village montagnard par rapport aux centres de la culture normale. Cette réponse est portée par une poétique qui sinsinue dans l'intimité des villageois pour suivre avec eux le chemin qui va de la terre familière au ciel idéal. Cette poétique est déjà une décision sur le statut de ces hérétiques. (...) la règle de méthode recouvre en fait une décision philosophique et politique, celle qui hiérarchise deux sortes de parole : celle qui fait acte et celle qui fait seulement indice. La parole des juges ou celle des théologiens fait acte. Celle des hérétiques de village, elle, fait seulement indice de la "culture du village". La distinction, ici encore, remonte à Aristote : il y a la parole (logos) qui met le juste et l'injuste en discussion et il y a la voix (phonè) qui exprime plaisir ou souffrance. C'est là le fond de la question sur laquelle le récit de l'historien décide : avant de savoir si les paroles peuvent par elles-mêmes attester le réel, il faut savoir qui parle et quelle parole est reçue comme l'expression d'une pensée $e^{30}$.

Ce que fait Carlo Ginzburg avec Menocchio, selon Rancière, c'est de rompre avec cette logique pour montrer la capacité des gens ordinaires à élaborer des idées extravagantes et à les soutenir jusqu'au bout ; cela va contre l'interprétation dominante de l'hérésie qui la ramène à la normalité d'une religion populaire. Cela ne demande pas seulement une grande attention pour percevoir le moment où la parole du sujet s'infiltre dans le discours de l'institution, cela requiert aussi une attitude volontaire, une prise de décision que l'on peut qualifier de politique : il s'agit de décider que Menocchio a une parole et pas seulement une voix.

Pratiquant ainsi une histoire en quête d'estrangement, il me semble que l'on s'éloigne d'une idée classique de la distance, celle que Carlo Ginzburg lui-même analyse dans un article sur la perspective, et dont le noyau conceptuel comporte l'idée de supériorité sur les vérités passées (la supériorité du verus Israël dans l'article en question). Passées, ces vérités seraient dès lors dépassées, moins vraies que notre vérité à nous qui nous considérons nous mêmes comme le produit de notre histoire ; une histoire qui serait celle de la réalisation de notre communauté (qu'elle soit celle d'un peuple élu, d'une nation ou d'une classe) et dont les historiens seraient chargés de mettre en lumière la logique interne, les lois cachées $^{31}$. Si cette histoire a dominé, c'est qu'elle s'est construite comme une parole institutionnelle dont les historiens étaient en quelque sorte des aèdes platoniciens chargés d'exprimer une idée qui leur venait d'en haut.

30 Jacques Rancière, « De la vérité des récits au partage des âmes », Critique, 2011/6, n 769-770, p. 474-484, citation à la p. 482.

31 "Distance et perspective. Deux métaphores ", in $A$ distance, op. cit., p. 147-164. 


\section{Conclusions}

À la fin de cet article, Carlo Ginzburg s'exclame : "Qui s'emparera de notre notion de l'histoire et la fera sienne, tout en en rejetant peut être le noyau conceptuel, exprimé par la métaphore de la perspective ? " ${ }^{32}$. La réponse pourrait être : Carlo Ginzburg lui-même. Pourquoi ne pas penser en effet que l'estrangement est précisément ce qui nous situe en dehors de ce noyau conceptuel de la perspective, ce qui nous permet de considérer les sujets d'hier comme aussi étranges que ceux d'avant-hier, mais vraiment étranges et non pas comme la réalisation presque parfaite de ce que nous sommes aujourd'hui (plus parfaite que ceux d'avant hier en tout cas) selon une logique de progrès continu. Une histoire où la vérité des discours des institutions et des producteurs de norme est passée au crible d'une vision conflictuelle qui n'est pas seulement celle de points de vue différents ou multiples. Le dialogue, le conflit des visions de la réalité n'est plus celui de deux ou plusieurs observateurs contemporains mais celui que l'historien organise entre le présent de ses propres catégories et le passé qu'il envisage : la vérité qu'il produit n'est pas, n'est plus, la vérité sur ce que nous sommes, supérieure à la vérité du peuple de l'ancienne alliance, mais la vérité sur ce que nous ne sommes pas.

L'historien, en allant chercher dans ce pays étranger qu'est le passé " ce que nous ne sommes plus, ce que nous ne sommes pas " rapporte cette étrangeté et la verse aux débats du présent. Il peut grâce à cette attitude tenir à égale distance la vieille perspective et l'identification émotive et nous rendre étranges à nous-mêmes, ou au moins nous rendre moins perméables aux discours institutionnels d'identification qui s'appuient sur le passé. En rendant étranges les identités de nos grands-parents, il peut enfin briser le cercle du récit du passé traumatique en créant de la distance entre les victimes et les souffrances passées, leur passé de souffrance, et effrayer ainsi les fantômes du passé familial.

François Godicheau

IUF

EA $4574 \mathrm{SPH}$

Université Michel de Montaigne Bordeaux 3

Francois.Godicheau@u-bordeaux3.fr 


\section{Résumé}

Cet article est une tentative d'utilisation de la notion d'estrangement pour l'histoire de périodes très récentes. Ces périodes présentent une combinaison de deux difficultés qui rendent la pratique de l'estrangement particulièrement nécessaire à l'écriture de l'histoire : d'une part, la force des politiques de mémoire, dont l'Espagne actuelle est un exemple saisissant, et d'autre part, une tendance à la naturalisation du passé liée à une conception linéaire de la notion de distance.

\section{Mots-clés}

Estrangement, histoire et mémoire, vérité, écritures du passé, Espagne.

\section{Abstract}

This article tries to improve the notion of straniamento for recent historical times. These times present a combination of two problems that make necessary the practice of straniamento in the writing of history: on a hand, the strength of the politics of memory, specially in Spain in the present, and on the other, an inclination toward the naturalisation of the past, due to a linear concept of distance.

\section{Keywords}

Estrangement effect, history and memory, truth, historiography, Spain. 